\title{
Research on Application of Spatial Information Technology in Gas Pipeline Repair
}

\author{
Ming Yong \\ CHENGDU RADIO and TV UNIVERSITY, Sichuan, Chengdu, China
}

1234@163.com

Keywords: GPS, GIS, booster, repair.

\begin{abstract}
With the application of spatial information technology in repair of gas networks, SCADA system is combined with GIS system to determine the damaged equipment and components of the booster, and the severity of the booster. The technical parameters including the manufacture, size, material, size and type of the damaged device and components is achieved by the database of gas networks. According to the geographical environment of the booster and pipeline inbuilt conditions, the machinery for repair, engineering technicians and the number is determined. According to the geographical environment of the booster and transportation network, GIS is used to make the shortest route analysis to computer the repairing route. And based on the technical parameters, the material department rapidly prepares the repairing materials for the repair personnel. Through the above measures, rapid repair is implemented.
\end{abstract}

\section{Introduction}

With the development of spatial information technology and the expansion of the technology at home and abroad, booster repair attracts the attention in China, but the technology is backward. The application of spatial information in the field is not reflected completely. The introduction of spatial information technique not only can improve the repair efficiency, but also can reduce the personnel, which can realize the automation of booster repair. The implementation model of the system is that the computer judges the booster condition by real-time analysis on the data of SCADA system, monitors the receiving information of the booster and makes booster analysis, and achieves the positioning of the booster. The information system of the pipe network inquires the database of the pipe network for achieving the tubular product and pipe diameter of accidental section, which achieves the required information of the repairing materials. By using GIS for the best route analysis, the best repairing route is achieved, and the complementary of GIS, pipe network information system and SCADA in technique is implemented lastly, which can replace the traditional repairing technique.

\section{Design Idea}

The system provides that the information system of pipe network determines the damaged device and components when the pipe network booster accident happens, and it is sent to the repair personnel with the form of pipe network repair report list, which realizes rapid and efficient repair. Telephone alarm determines the location of the booster, and the mouse is used to click the pipeline of the booster in pipe network information system, which can show the influenced section area. And the manufacture, size, material, size and type of the damaged device and components is achieved by the database of pipe networks. According $t$ the geographical environment and pipeline inbuilt condition, the influenced section area is reflected under the support of SCADA system. And it shows the important information of the users, and the repair machinery, engineering technical staff and the number is determined. After the system prints and transmits the repairing report form, the repair personnel receives the accepts the repairing report form rapidly and informs the reserve personnel. Achieving the best route under the technical support of GIS is necessary to improve repair. 
SCADA system positions booster. SCADA system is used for data acquisition and monitoring pipe system. Gas pipeline operates based on SCADA system. The system judges the operation state of the pipeline according to the acquired data and the knowledge of the urban gas pipeline, for realizing operation and daily management of pipelines. SCADA system can realize the collection of all fields and RTU parameters and equipment control. There are many methods of detecting oil-gas pipeline leakage, and the reliability is high. However, the specific leakage position instrument can mark according to the mileage and the distance of a station yard and high spot, which is difficult to accurately determine the geographical condition of the leakage point. Therefore, commanding the maintenance and repair of the booster was only based on experience judgment before. The way of combining GIS technique can solve the above problems. The technique can evidently show the coordinator of the leakage point, compute the leakage rate, analyze the geographical condition of the leakage point, for judging the severity of the accidents. The technical support and the solution for the repair schema is sent to the pipeline information system by the way of information.

Pipe network information system achieves material parameters. The material type of the booster positioned by SCADA system and the manufactures, size, materials and type of the damaged device and components is achieved by pipe network information system. According to the geographical environment and inbuilt condition of the pipelines, the repair machinery, engineering technical personnel and number is determined, which reduces the intensity of production and regulating personnel.

Acquisition of the best route. Pipe network information system sends the materials of pipelines to the personnel of the storage room for providing materials for the repair personnel. When the pipeline accident is repaired, GIS and GPS is combined. On one hand, GPS system is used to accurately achieve the position and the traffic condition, and transfers the data to GIS in real time, for computing the best route. On the other hand, multi-source annular spacial data model is used for network trace analysis. Breadth first search algorithm is used to traverse the digraph and search out the control valve, and trace the directed digraph for rapidly determining faulty section. The influencing range and influencing degree of the accidents is analyzed to simulate the dynamic change of the pipe network, for generating repair scheme. The pipeline drawing of the repair area and user information is output for scheduling the facility of pipe network and implementing repair. It is evident that the above technical scheme fully reflects the high efficiency of modern technique compared with traditional accident process.

\section{Implementation of System}

System interface. The pipelines and gas devices are displayed in classification with the form of tree directory in pipe network equipment list of the system, which is easy for the users to inquire and operate the information. Information display area is divided into two label pages which show pipelines or detailed information of devices and figures of pipe networks. Pipe network graph and data display area is divided into two label pages which show pipe network graph and the data table of pipelines and devices. The system coordinator of the present mouse in pipe network graph is shown in status bar.

1. Position of booster determines to achieve parameters

Telephone alarm the position of booster. Using mouse to click pipelines of booster in pipe network information system can show the influenced section area. The technical parameters including manufacture, size, materials and type of the damaged device and components is achieved by pipe network database. And the attributes of the parameters are sent to the repair report table of pipelines, and the table is transfers to the technical department.

2. Technical department takes repairing measure to achieve pipeline repair report form

The internal network of the system achieves the repair report form. The repair report form is detected if there is technical failure. After ensuring that the report form is right, the repair scheme is determined to prepare repair machinery, engineering technicians and number. And the material preparation department is informed to prepare materials. 
3. GPS is used to achieve the shortest route The commonest shortest route algorithms of GPS research include ant colony algorithm and Jester algorithm. And the idea of ant colony algorithm is used in the paper, setting presetting points in main streets of the map, after determining the coordinator of the starting point and the destination, the presetting point of two points is found out according to the coordinate difference of two points for finding out the optimal value of the adjacent presetting points, and finding the shortest route at last. Installing vehicle GPS in recovery vehicles can rapidly achieve the accident spot for completing repair.

Implementation of system progress. The software for urban gas pipe-broken analysis of MapXtreme includes Visual Studio 2005, MapXtreme2008 and MapInfo Professional. The function of Visual Studio 2005 is to develop environment, that of MapXtreme2008 is to develop component library and that of MapInfo Professional is to establish topology relationship of urban gas pipe network.

(2) Design out-of-warehouse management module of maintenance materials. In the implementation of spatial information technology, out-of-warehouse management module of maintenance materials is the emphasis of the system. The implementation model is starting Visual Studio 2005, clicking the start menue, choosing Micosoft Visual Studio 2005, establishing new project, clicking file-establishment-project and choosing MapXtreme 6.8.0 in term types, choosing MapXtreme 6.8.0 MapForm application in template.

(3) Adding the button of opening pipe network graph in Form 1. Clicking $[\mathrm{ab}$ in all Windows forms on the left toolbar, and changing Text button1 of the attributes into opening pipe network graph. 1Function analysis. Out-of-warehouse management of materials is to complete the out-of-warehouse function of materials. After the users extract the materials, the number reduces. Therefore, out-of-warehouse management of materials not only needs to record the number and type of the delivery of the users, but also requires to modify the information of materials and the number of the materials in the form. In the system, the function is implemented by calling trig_outGoods.

Form design. Window quotient filed is established, and is named as frmOSManage.cs. And it is used to implement out-of-warehouse management function of materials. The controls, attribute setting of controls and other usages of the form are as shown in the form. 
Table 1. Controls and attribute setting of out-of-warehouse management form

\begin{tabular}{|c|c|c|c|}
\hline Control type & Space name & Attribute setting & Usages \\
\hline \multirow[t]{7}{*}{ TextBox } & txyOSNum & $\begin{array}{l}\text { Setting ReadOnly attribute } \\
\text { to be False }\end{array}$ & $\begin{array}{l}\text { out-of-warehouse } \\
\text { number }\end{array}$ \\
\hline & txtGOPrice & $\begin{array}{l}\text { Setting ReadOnly attribute } \\
\text { to be true }\end{array}$ & Unit price of materials \\
\hline & txtGSPrice & $\begin{array}{l}\text { Setting ReadOnly attribute } \\
\text { to be False }\end{array}$ & $\begin{array}{lcc}\text { Total amount } & \text { of } \\
\text { out-of-warehouse }\end{array}$ \\
\hline & txtospeople & $\begin{array}{l}\text { Setting ReadOnly attribute } \\
\text { to be False }\end{array}$ & Forwarder \\
\hline & txtOSUnit & $\begin{array}{l}\text { Setting ReadOnly attribute } \\
\text { to be False }\end{array}$ & Delivery agent \\
\hline & txtHPeople & $\begin{array}{l}\text { Setting ReadOnly attribute } \\
\text { to be False }\end{array}$ & Sponsor \\
\hline & txtOSRemark & $\begin{array}{l}\text { Setting ReadOnly attribute } \\
\text { to be False }\end{array}$ & Notes \\
\hline \multirow[t]{4}{*}{ ComboBox } & CboxSName & $\begin{array}{l}\text { Setting ReadOnly attribute } \\
\text { to be False }\end{array}$ & Name of warehouse \\
\hline & cboxGName & $\begin{array}{l}\text { Setting ReadOnly attribute } \\
\text { to be False }\end{array}$ & Name of materials \\
\hline & cboxGSpec & $\begin{array}{l}\text { Setting ReadOnly attribute } \\
\text { to be False }\end{array}$ & $\begin{array}{ll}\text { Specification } & \text { of } \\
\text { materials } & \\
\end{array}$ \\
\hline & cboxGUNIT & $\begin{array}{l}\text { Setting ReadOnly attribute } \\
\text { to be False }\end{array}$ & Unit of measurement \\
\hline \multirow[t]{3}{*}{ Button } & btnAdd & $\begin{array}{l}\text { Setting TextImageRelation } \\
\text { attribute to be } \\
\text { TexBeforImage, and setting } \\
\text { ImageAlignattribute to be } \\
\text { MiddleLeft }\end{array}$ & Out-of-warehouse \\
\hline & btnDel & $\begin{array}{l}\text { Setting TextImageRelation } \\
\text { attribute to be } \\
\text { TexBeforImage, and setting } \\
\text { ImageAlignattribute to be } \\
\text { MiddleLeft }\end{array}$ & Delete \\
\hline & btnExit & $\begin{array}{l}\text { Setting TextImageRelation } \\
\text { attribute to be } \\
\text { TexBeforImage, and setting } \\
\text { ImageAlignattribute to be } \\
\text { MiddleLeft }\end{array}$ & Exit \\
\hline DataGridview & dgvOSManage & $\begin{array}{l}\text { Setting ReadOnly attribute } \\
\text { to be True }\end{array}$ & $\begin{array}{l}\text { Showing } \\
\text { out-of-warehouse } \\
\text { information of materials }\end{array}$ \\
\hline
\end{tabular}

\section{Conclusions and Precautions}

In the existing developed pipe network systems, the important GIS analysis function is booster analysis. Combined with the function of GPS, by booster analysis of urban gas pipe networks, the attributes of pipelines, repair materials and the shortest route information of the accident spot can be displayed with the digital form when there are accidents, which can efficiently provide information for repair personnel, and reduce the property loss and improve repair efficiency. The booster analysis method in the paper is based on the requirement of booster repair to complete the related programming, for improving efficiency of booster repair.

\section{References}

[1] Meng Xiao, Research on water supply pipeline booster accident based on Super Map.

[2] Mo Shanjun, Feng Qimin, Wu Qinmin, Technical implementation of gas pipe network accident valve-turnoff analysis under ArcInfo platform [J], Computer Application and Software, 007-2,24(2), 91-94.

[3] Li Pei, He Zhongshi, Design and implementation of facility management system based on ArcGIS and GPS, 2009. 
[4] He Fang, Analysis method of water supply pipeline booster analysis method and research on preventive technique, Shanghai, Tongji University, 2004.

[5] g-tsung Chang, Introductory theory of geographical information system (Chen Jianfei), Beijing, Tsinghua University Press, 2009.358 375.

[6] Liu Yanhui, Zhang Haiying, Application of GPS technique in water-supply pipeline repair. 